\title{
Les effets inattendus des expériences de recombinaison homologue
}

\author{
Matthieu Lévi-Strauss
}

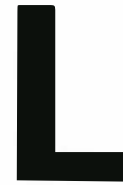

a génétique moléculaire nous avait jusqu'à présent habitués à une vision très cohérente de la biologie où les approches multiples d'un même problème convergeaient toutes vers le même mécanisme simple. Ainsi, par exemple, une fois clonés les gènes codant pour l'hormone de croissance, le GH-RH, et leurs récepteurs, on s'aperçut que, comme prévu, des nanismes héréditaires pouvaient être dus, chez l'homme ou chez la souris, à la mutation de gènes codant pour l'une ou l'autre de ces protéines. De façon toujours aussi prévisible, les déficits en hormone de croissance peuvent être compensés par l'administration de cette protéine produite par des bactéries recombinantes transformées par le gène humain, lequel est d'ailleurs également capable de faire grandir de façon spectaculaire les souris transgéniques qui le surexpriment.

De la même façon, les maladies héréditaires sont souvent, mais comme on le verra plus bas, pas toujours, dues à des mutations dont l'effet prévisible correspond au phénotype que l'on observe effectivement. C'est, par exemple, le cas de l'hémophilie qui est provoquée par l'altération de facteurs de la coagulation, et du daltonisme où, comme l'on pouvait s'y attendre, certaines protéines photoréceptrices ne sont plus fonctionnelles.

Cependant, les choses se compliquent quand, au lieu d'observer l'effet de mutations auxquelles on s'est justement intéressé parce qu'elles provoquaient une maladie, on étudie les conséquences de l'inactivation, par recombinaison homologue, de gènes dont on tente ainsi de confirmer la fonction présumée. Très curieusement, les expériences de ce type ont souvent un effet totalement imprévu, ou bien n'ont même parfois aucun effet visible. Comme je le signalais il y a quelques années [1], les souris dépourvues de c-src ne souffrent que d'une ostéopétrose alors que leurs neurones et leurs plaquettes qui, normalement, expriment le plus cette protéine sont tout à fait normaux $\left(m / s n^{\circ} 5\right.$, vol. 7, p. 509). De très nombreux exemples sont depuis venus renforcer l'idée qu'il est le plus souvent quasiment impossible de prédire les effets de l'inactivation des gènes, même s'ils codent pour des protéines très étudiées dont on pensait bien connaître la fonction. Ainsi, les souris dépourvues de $\mathrm{N}$ CAM, dont de très classiques expériences ont pourtant montré le rôle crucial dans l'adhérence entre neurones embryonnaires, ou entre neurones et cellules musculaires [2], paraissent normales, à part une légère diminution de la taille de leurs bulbes olfactifs et de leurs performances dans des tests de mémoire spatiale [3]. La modestie des effets de l'inactivation du gène codant pour la synapsine I est elle aussi surprenante [4]. Plusieurs articles de grande qualité ont, en effet, mis en évidence un rôle de cette protéine dans la libération des neurotransmetteurs au cours de laquelle sa phosphorylation était censée permettre aux vésicules synaptiques de se détacher des filaments d'actine du cytosquelette, le long desquels elles sont parquées lorsque les neurones sont au repos. Pourtant, les souris qui en sont dépourvues ne se distinguent des animaux normaux que par l'augmentation d'un paramètre qui mesure in vitro le renforcement de la réponse d'une synapse lors de sa deuxième stimulation. En outre, cet effet semble tout à fait inattendu, si l'on en juge par la complexité des explications proposées par les auteurs de l'article. Le cas de l'enzyme neuronale productrice de monoxyde d'azote est également très étonnant puisque son inactivation, qui n'a pas d'effet dans le système nerveux central malgré les nombreux rôles que ce gaz était censé y jouer, provoque une surprenante hypertrophie du sphincter du pylore, à l'origine d'une dilatation de l'estomac [5]. Il suffit de feuilleter les volumes récents des grandes revues scientifiques pour trouver de nombreuses autres expériences de recombinaison homologue aux effets imprévus ou modestes. Je citerai encore les cas de l'antigène lymphocytaire CD2 [6] ou de la ténascine [7] sans lesquels les souris vivent fort bien.

Les mutations naturelles sont, elles aussi, parfois très surprenantes puisqu'on ignore encore la physio- 
pathologie moléculaire de plusieurs maladies héréditaires dont les gènes responsables ont pourtant déjà été identifiés. C'est, par exemple, le cas étonnant de certaines maladies qui sont dues à la mutation d'un gène codant pour une protéine ubiquiste et qui ne touchent paradoxalement qu'un tissu très particulier. Ainsi, les déficits en adénosine désaminase ou en purine nucléoside phosphorylase, deux enzymes ubiquiste qui interviennent dans le métabolisme des bases puriques, ont l'air d'être sélectivement toxiques pour les lymphocytes et provoquent des déficits immunitaires [8]. De la même façon, certaines formes familiales dominantes de sclérose latérale amyotrophique, dans lesquelles seuls les neurones moteurs sont touchés, sont dues à des mutations du gène codant pour la très ubiquiste superoxyde dismutase [9]. Enfin, la maladie de Leber, due à une mutation mitochondriale homoplasmique, est à l'origine d'une cécité par dégénérescence des neurones qui forment le nerf optique [10]. Cette dernière observation est d'autant plus étonnante que d'autres mutations mitochondriales, hétéroplasmiques cette fois, mais dont on pourrait imaginer qu'elles ont le même effet délétère et ubiquiste sur le métabolisme énergétique, touchent sélectivement des tissus aussi différents que le muscle ou le cerveau [11].

Parmi toutes les curiosités qui viennent d'être décrites, certaines peuvent sans doute s'expliquer simplement si l'on remet en cause, comme l'a proposé Erickson dans un élégant article, l'intérêt biologique de l'étude de la spécificité tissulaire d'expression des gènes [12]. Ainsi, l'expression de c-src dans les neurones et dans les plaquettes, plutôt que le reflet d'un rôle de la protéine dans ces cellules, n'est peutêtre que simplement tolérée car dépourvue de toute conséquence fonctionnelle. La même notion peut être également invoquée, sous une forme un peu différente, pour expliquer que la diminution de l'ordre de $50 \%$ de l'activité de la superoxyde dismutase qu'on observe dans les scléroses latérales amyotrophiques familiales soit sans consé- cellulaires qui produiraient ordinairement cette enzyme en excès. Les neurones moteurs pourraient être particulièrement vulnérables à une baisse de l'activité de leur système de détoxification des radicaux libres et seraient ainsi les seules cellules affectées par la mutation.

La redondance est l'explication la plus couramment proposée pour rendre compte de la modestie des effets de certaines expériences de recombinaison homologue. Selon cette hypothèse, l'absence d'effet de l'inactivation d'un gène indiquerait simplement que le rôle présumé de la protéine pour laquelle il code pourrait être tenu par une autre protéine, susceptible de la remplacer. Outre son caractère tautologique, cette explication ne rend pas compte de la forte conservation phylogénétique de certaines protéines qu'on attribue habituellement à l'existence d'une importante pression de sélection, mais qui paraît difficile à concilier avec la viabilité apparente des souris chez lesquelles les gènes correspondants sont rompus. Il faut toutefois reconnaître que l'observation d'animaux dans les conditions artificielles du laboratoire ne permet pas de bien estimer les effets réels d'une mutation au cours de la vie sauvage durant laquelle la pression de sélection s'exerce effectivement. Cependant, on ne peut qu'être frappé par l'absence de corrélation entre le degré de conservation phylogénétique d'une protéine et l'importance des effets délétères provoqués par son inactivation. On aurait en effet pu s'attendre à ce que la rupture des gènes les plus conservés entraîne les effets les plus dramatiques, comme les morts in utero que l'on observe parfois. Pourtant, la suppression de la protéine En2, dont un segment long d'une centaine d'acides aminés est quasiment identique à son homologue de poisson-zèbre [13] et ne diffère que de $27 \%$ de son homologue de drosophile [14], ne provoque qu'une légère diminution de la taille du cervelet qui semble n'avoir aucune conséquence fonctionnelle [15].

Tout comme les manipulations génétiques des organismes vivants, les manipulations pharmacologiques sont aussi parfois imprévisibles. Qui aurait pu, par exemple, prévoir l'usage thérapeutique antiinflammatoire des corticoïdes en connaissant seulement les effets transcriptionnels pléiotropes induits par la liaison de ces hormones à leur récepteur ubiquitaire? Tout aussi étonnant est l'exemple de la colchicine qui, bien qu'inhibant la polymérisation de la très ubiquiste tubuline, traite pourtant à merveille les crises de goutte en agissant probablement de façon relativement spécifique sur les cellules phagocytaires.

Cette imprévisibilité, dont on vient de voir quelques exemples, est d'autant plus étonnante qu'elle n'est pas générale et que, heureusement, la biologie nous permet souvent de bien comprendre le fonctionnement des êtres vivants. Cependant, l'existence de ces curiosités doit inciter les biologistes à tenter de remettre en cause la vision qu'ils ont de leurs objets d'étude qui ne sont pas issus de la technologie, et qui n'ont donc aucune raison de fonctionner comme des machines dans lesquelles chaque pièce a un rôle bien défini. Notre situation ressemble à celle des physiciens de la fin du siècle dernier qui, à côté de réussites éblouissantes qui permirent un énorme essor technologique, étaient confrontés à une accumulation de problèmes apparemment insolubles. Ce n'est peut-être qu'au prix d'un bouleversement comparable à celui que Planck et Einstein infligèrent à la physique du début de notre siècle que la biologie de demain pourra enfin prédire le résultat des expériences de recombinaison homologue

\section{RÉFÉRENCES}

1. Lévi-Strauss M. Striptease génétique chez les souris. La Recherche 1991; 22: 1230-1.

2. Rutishauser U, Grumet M, Edelman GM. Neural cell adhesion molecule mediates initial interactions between spinal cord neurons and muscle cells in culture. $J$ Cell Biol 1983; 97 : 145-52.

$$
\mathrm{m} / \mathrm{s} n^{\circ} 5 \text { vol. 10, mai } 94
$$


3. Cremer H, Lange R, Christoph A, Plomann M, Vopper G, Roes J, Brown R, Baldwin S, Kraemer P, Schefff S, Barthels D, Rajewsky K, Wille W. Inactivation of the NCAM gene in mice results in size reduction of the olfactory bulb and deficits in spatial learning. Nature 1994 ; 367 : 455-9.

4. Rosalh TW, Geppert M, Spillane I), HerzJ, Hammer RE, Malenka RC, SüdhofTC. Short-term synaptic plasticity is altered in mice lacking synapsin 1. Cell 1993 ; 75 : 661-70.

5. Huang PI, Dawson TM, Bredt ISS, Snyder SH, Fishman MC. Targeted disruption of the neuronal nitric oxide synthase gene. Cell 1993; 75 : 1273-86.

6. Killeen N, Stuart SG, Littman IR. Development and function of $\mathrm{T}$ cells in mice with a disrupted CI)2 gene. LMBOO J 1992 ; 11: 4329-36.

7. Saga Y, Yagi T, IkawaY, Sakakura T, Aizawa S. Mice develop normally without tenascin. (ienes Dev 1992; 6: 1821-31.

8. Scriver CR, Beaudet AI, Sly WS. Valle I). The metabolic basis of inherited disease. Sixth Edition, Mc Graw-Hill, 1989.

9. Deng HX, Hentati A, Tainer JA, et al. Amyotrophic lateral sclerosis and structural defects in $\mathrm{Cu}, \mathrm{Zn}$ superoxide dismutase. Science 1993 ; 261 : 1047-51.

10. Wallace I)(, Singh G, Lott MT Hodge JA, SchurrTG, Lezza AMS, Elsas II L.J, Nikoskelainen EK. Mitochondrial IDNA mutation associated with I.eber's hereditary optic neuropathy. Science 1988 . 242: 1427-30.

11. Dreyfus JC. L Les maladies du génome mitochondrial. médecine/sciences 1991; 7 : 172-4

12. Erickson HP. Gene knockouts of c-src, transforming growth factor $\beta 1$ and tenascin suggests superfluous, nonfunctional expression of proteins. J Cell Biol 1993 120 : 1079-81.

13. Fjose A, Eiken HG, Njolstad PR, Molven $A$, Hordvik I. A zebrafish engrailed-like homeobox sequence expressed during embryogenesis. FEBS lett 1988; 231: 355-60.

14. Joyner AL, Martin GR. lin-l and lin-2, two mouse genes with sequence homology to the drosophila engrailed gene: expression during embryogenesis. Genes Dev $1987 ; 1: 29-38$.

15. Joyner AL, Herrup K, Auerbach BA, Davis CA, Rossant J. Subtle cerebellar phenotype in mice homozygous for a targeted deletion of the En-2 homeobox. Science $1991 ; 251: 1239-43$

\section{Lévi-Strauss}

Chargé de recherche, Inserm U. 114, Collège de France, 11, place Marcelin-Berthelot, 75005 Paris, France.

TIRÉS A PART

M. I .évi-Strauss.

$\mathrm{m} / \mathrm{s} n^{\circ} 5$ vol. 10 , mai 94 